\title{
Überlegungen zur Rechtsgrundlage des Bußgeldverfahrens der Europäischen Kommission im Kartellrecht nach dem Vertrag von Lissabon
}

Ich möchte hier einige Überlegungen zur Problematik der Rechtsgrundlage des Bußgeldverfahrens der Europäischen Kommission im europäischen Kartellrecht anstellen.

Die Tätigkeit der Kommission zur Ahndung der Verstöße gegen den freien und fairen Wettbewerb ist in den letzten zwei Dekaden wichtiger geworden, was sich nicht zuletzt an der immer stärker wachsenden Höhe der verhängten Bußgelder zeigt: erreichte die Höhe eines einzelnen Bußgeldes zu Beginn der 90er Jahre kaum die Summe von einigen Millionen Euro (bzw. in den alten Währungen), so hat die Höhe eines Bußgeldes in den letzten Jahren bereits eine Milliarde Euro überschritten. ${ }^{1}$

Zwar darf die Höhe der Bußgelder aus rein theoretischer Sicht keinen Einfluss auf die Beurteilung des dazu führenden Verfahrens haben - ein Bußgeldverfahren muss immer auf eine gültige und den rechtsstaatlichen Anforderungen genügende Rechtsgrundlage gestützt sein und den Erfordernissen der Fairness und der Grundrechte entsprechen, unabhängig davon, ob die Höhe des Bußgeldes 500 Euro oder 500 Millionen Euro beträgt - doch lenkt gerade die Höhe der Bußgelder die öffentliche und juristische Aufmerksamkeit auf echte oder vermeintliche Schwächen des dazu führenden Verfahrens. $^{2}$

Im Folgenden möchte ich nur einen Kritikpunkt - das Problem der Rechtsgrundlage des Bußgeldverfahrens - aufgreifen und meine rein persönliche Sicht dazu als einen Beitrag zur Weiterführung der juristischen Diskussion kurz darlegen.

\section{Die Rechtsgrundlage}

Die Rechtsgrundlage für das Recht der Kommission, Bußgelder zu verhängen, ist Art. 23 Abs. 2 der Verordnung Nr. 1/2003, ${ }^{3}$ der vorsieht, dass die Kommission berechtigt ist, Bußgelder gegen die beteiligten Unternehmen zu verhängen, wenn diese vorsätzlich oder fahrlässig gegen die Wettbewerbsregeln der Art. 101 oder 102 des AEUV (vormals Art. 81 und 82 des EG-Vertrages) verstoßen. Die Tatbestandsmerkmale der Verstöße sind im Art. 23 Abs. 2 detailliert und genau formuliert, während auf der Rechtsfolgeseite bei der Bemessung der Höhe des Bußgeldes bis zu der festgesetzten Maximalhöhe der Kommission ein weiter Ermessenspielraum eingeräumt ist. Die maximale Höhe des

1 Vgl. Marco Mansdörfer, Sven Timmerbeil: Das Modell der Verbandshaftung im europäischen Kartellbußgeldrecht. Europäische Zeitschrift für Wirtschaftsrecht, 2011, S.214.

2 Umfassend s. Jürgen Schwarze, Wolfgang Bosch, Rainer Bechtold: Rechtsstaatliche Defizite im Kartellrecht der Europäischen Gemeinschaft. Eine kritische Analyse der derzeitigen Praxis und Reformvorschläge. Gleiss Lutz Rechtsanwälte 2008.

3 Verordnung (EG) v.16.2.2002 zur Durchführung der in Art. 81 und 82 niedergelegten Wettbewerbsregeln, ABl. L Nr.1, 4.1.2003, S.1. 
Bußgeldes ist auf $1 \%$ des Gesamtumsatzes im vorausgegangenen Geschäftsjahr für leichte Verstöße und auf $10 \%$ für andere Verstöße beschränkt. Art. 23 Abs. 3 besagt, dass bei der Bemessung der Geldbuße die Dauer und die Schwere des Verstoßes zu berücksichtigen ist.

Den recht weiten Spielraum bei der Bemessung der Bußgeldhöhe hat die Kommission aber selbst eingeschränkt, in dem sie die Leitlinien für das Verfahren zur Festsetzung der Bußgelder erlassen hat. ${ }^{4}$ Im Unterschied zu den Bestimmungen der Verordnung, legen die Leitlinien detailliert in 38 Punkten dar, wie die Kommission die Bußgelder festsetzt. Die Bußgeldberechnung geschieht in zwei Stufen. Zuerst wird ein Grundbetrag festgesetzt, dabei wird der Grundbetrag im Verhältnis zum Umsatz mit den vom wettbewerbsrechtlichen Verstoß betroffenen Waren berechnet. Im zweiten Schritt wird der Grundbetrag dann anhand von erschwerenden oder mildernden Umständen angepasst.

\section{Die Bestimmtheit der Rechtsgrundlage}

Würde Art. 23 Abs. 2 und Abs. 3 der Verordnung Nr.1/2003 alleine zur Anwendung kommen, so würde dies aus der Sicht der rechtsstaatlichen Bestimmtheit der Regelung äußerst problematisch sein. Die konkrete Auskunft darüber, wie diese Vorschrift auf dem Einzelfall angewendet werden soll, geben aber die Leitlinien. Die Rechtsgrundlage für die Festsetzung der Bußgelder erschließt sich inhaltlich vollständig nur dann, wenn beide Akte zusammen gelesen werden.

Die Leitlinien der Europäischen Kommission gehören allerdings nicht zu den formell verbindlichen Rechtsakten der Union, sondern zu den sogenannten „soft-law“ Instrumenten des Unionsrechts. ${ }^{5}$ Die Leitlinien sind keine Rechtsnormen im eigentlichen Sinne, sondern Verhaltensnormen, die dennoch eine rechtliche Außenwirkungen entfalten, indem sie einen Hinweis auf die zu befolgende Verwaltungspraxis enthalten, von der die Verwaltung im Einzelfall nicht ohne Angabe von Gründen abweichen kann. ${ }^{6}$ Die Gründe für die Abweichung müssen triftig sein und insbesondere den Gleichbehandlungsgrundsatz beachten. ${ }^{7}$

Die Leitlinien stellen also eine Selbstverpflichtung der Kommission hinsichtlich der Ausübung ihr durch Art. 23 Abs. 2 und 3 der Verordnung Nr.1/2003 eingeräumten Ermessens dar. Diese in den Leitlinien beschriebene Selbstverpflichtung der Kommission, ihr Ermessen im bestimmten Sinne auszuüben, begründet schützenswertes Vertrauen des Adressaten und ist daher für die Kommission selbst bindend. ${ }^{8}$ Die Einhaltung der Leitlinien ist daher durch die Gerichte der Union überprüfbar. ${ }^{9}$

4 Leitlinien für das Verfahren zur Festsetzung von Geldbußen gemäß Artikel 23 Abs. 2 Buchstabe a) der Verordnung (EG) Nr.1/2003, ABl. C 210, 1.9.2006, S.2.

5 Jürgen Schwarze: Soft Law im Recht der Europäischen Union. Europarecht, 2011, S.10.

6 Urteil des EuGH in der Rechtssache C-397/03 P v. 18.6.2006, Archer Daniels Midland, §91; vgl. in diesem Sinne auch das Urteil in den Rechtssachen C-189/02 P, C-202/02 P, C-205/02 P bis C-208/02 P und C-213/02 P v. 28.6.2005, Dansk Rørindustri, §§ 209, 210.

7 Ebenda.

8 Vgl. Thomas von Danwitz: Europäisches Verwaltungsrecht. Berlin 2008, S.252.

9 Vgl. Urteil des EuGH in der Rechtssache C-125/07 v. 24.9.2009, Erste Group Bank, §143. 
Aus der Sicht des Unternehmens ist diese „, Gesamtkonstruktion“ der Rechtsgrundlage, bestehend aus Art. 23 Abs. 2 und 3 der Verordnung Nr.1/2003 und die detaillierten Klarstellungen in den Leitlinien der Kommission, ${ }^{10}$ aber bestimmt genug, um den rechtsstaatlichen Anforderungen an die Bestimmtheit einer Regelung zu entsprechen. ${ }^{11}$ Auf jedem Fall sind die maßgeblichen Eckpunkte für die Berechnung und die Höhe der Bußgelder sichtbar. ${ }^{12}$ Insoweit gibt es keinen Platz für Bedenken, die nicht so einfach von der Hand zu weisen wären, wenn man die Regelung des Art. 23 Abs. 2 und 3 der Verordnung Nr.1/2003 alleine betrachten würde. ${ }^{13}$ Die Tatsache, dass sich der volle Inhalt dieser „Gesamtkonstruktion“ der Rechtsgrundlage erst aus zwei Akten mit unterschiedlichem Charakter erschließt, ist für die Beurteilung der Bestimmtheit der Regelung aus der Sicht der Adressaten - worauf es hier ankommt - irrelevant.

Neben der Berechnung des Bußgeldbetrags hat die Kommission auch andere wesentliche Aspekte des Verfahrens durch Mitteilungen festgesetzt.

Zum Beispiel die Einführung - in der Praxis sehr wichtigen - „Kronzeugen-Regelung" erfolgte durch die Mitteilung der Kommission über den Erlass und die Ermäßigung von Geldbußen in Kartellsachen. ${ }^{14}$ Auch das Akteneinsichtsrecht ist im wesentlichen durch die Mitteilung der Kommission über die Regeln für die Einsicht in Kommissionsakten in Fällen einer Anwendung der Artikel 81 und 82 EG-Vertrag, Artikel 53, 54 und 57 des EWR-Abkommens und der Verordnung (EG) Nr. 139/2004 detailliert festgelegt. ${ }^{15}$

Die Mitteilungen der Kommission sind wie die Leitlinien - soweit ausnahmsweise keine Ermächtigung zu ihrem Erlass gegeben ist - Instrument der „soft-law“, welches nur über das künftige Verhalten der Kommission informiert und sind daher im Prinzip nicht als Rechtsnormen einzuordnen. Sie können jedoch schutzwürdiges Vertrauen der Adressaten erzeugen und ihre Einhaltung bzw. Nichtigkeit kann im Einzelfall auch von den Gerichten der Union überprüft werden. ${ }^{16}$

Auch hier geben die Mitteilungen für die Betroffenen eine detaillierte Auskunft über die genannten Aspekte des Bußgeldverfahrens, woraus sie mit hinreichender Bestimmtheit entnehmen können, wie die Kommission ihr im Art. 23 Abs. 2 und 3 der Verordnung Nr.1/2003 eingeräumtes Ermessen ausüben wird.

Zusammenfassend ist festzustellen, dass die Regelung des Bußgeldverfahrens aus der Sicht der Betroffenen den rechtsstaatlichen Anforderungen an ihre Bestimmtheit genügt.

10 Vgl. Walter Frenz: Handbuch Europarecht. Band 2. Europäisches Kartellrecht. Berlin 2006, S.590.

11 Vgl. Urteil der EuGH in der Rechtssache C-266/06 v. 28.5.2008, Evonik Degussa, $\S 55$ ff.; Alfred Dittrich: Geldbußen im Wettbewerbsrecht der Europäischen Union. Zentrum für Europäisches Wirtschaftsrecht. Vorträge und Berichte, Nr.182, 2010, S.9 f.

12 Walter Frenz: Handbuch Europarecht. Band 2. Europäisches Kartellrecht. Berlin 2006, S.589.

13 A.A. aber Jürgen Schwarze: Europäische Kartellbußgelder im Lichte übergeordneter Vertrags- und Verfassungsgrundsätze. In: Europarecht, 2009, S.185 f.

14 ABl. C Nr.298, 8.12.2006, S.17.

15 AB1. C Nr.325, 22.12.2005, S.7.

16 Vgl. Ines Härtel: Handbuch Europäische Rechtsetzung. Berlin 2006, S.295 ff. 


\section{Die Rechtsgrundlage aus der Sicht des Demokratieprinzips}

Es wird jedoch kritisiert, dass eine solche „Gesamtkonstruktion“ der Rechtsgrundlage - prinzipielle Ermächtigung der Kommission zur Verhängung der Bußgelder mit vagen inhaltlichen Bestimmungen, während die Höhe des Betrages und wesentliche Aspekte des Verfahrens durch Leitlinien und Mitteilungen der Kommission, die keinen Rechtsnormcharakter haben, klargestellt sind - gegen das im Unionsrecht verankerte Demokratieprinzip verstößt. ${ }^{17}$

Das Demokratieprinzip - trotz der Kritik am echten oder angeblichen „Demokratiedefizit" - ist ein tragender Grundsatz der Europäischen Union. ${ }^{18}$ Ein Ausfluss des Demokratieprinzips ist Art. 290 Abs. 1 U Abs. 2 S.2 AEUV. Danach sind ,, die wesentlichen Aspekte eines Bereichs dem Gesetzgebungsakt vorbehalten “.

Gesetzgebungsakte sind aber gem. Art. 288 AEUV nur solche Akte der Union, die in einem formellen Gesetzgebungsverfahren - in der Regel durch den Rat und das Parlament - angenommen werden. Zwar können nach Art. 290 Abs. 1 S.1 und 2 in Gesetzgebungsakten der Kommission die Befugnis übertragen werden, Rechtsakte ohne Gesetzescharakter mit allgemeiner Geltung zur Ergänzung oder Änderung bestimmter nicht wesentlicher Vorschriften des betreffenden Gesetzgebungsaktes zu erlassen. In den betreffenden Gesetzgebungsakten müssen aber die Ziele, Inhalt, Geltungsbereich und Dauer der Befugnisübertragung ausdrücklich festgelegt werden.

Die neue Regelung des Art. 290 Abs. 1, die ohne Vorbild im bisherigen Vertrag ist, fasst die bisherigen sekundärrechtlichen Regelungen, Praktiken, in der Rechtsprechung und Theorie entwickelten Grundsätze zusammen, und verleiht dem Demokratieprinzip durch die ausdrückliche Kodifizierung auf der Ebene des Primärrechts eine neue Qualität. Das Ziel dieser neuen Bestimmung des Vertrages ist, das Demokratieprinzip stärker $\mathrm{zu}$ betonen und die Verantwortung des demokratisch stärker legitimierten Unionsgesetzgebers zu erhöhen. ${ }^{19}$ Das entspricht der Tendenz der Entwicklung der Union von einer Wirtschafts- zu einer weitergreifenden politischen Union.

Hinsichtlich der Leitlinien und Mitteilungen, die die Berechnung der Höhe der Bußgelder und das Verfahren näher festsetzen, stellt sich zunächst die Frage, ob sie den neuen - deutlicheren - Anforderungen des Demokratieprinzips, wie es jetzt in Art. 290 Abs. 1 AEUV ausgestaltet ist, nach dem Inkrafttreten des Vertrags von Lissabon Rechnung tragen sollten.

Hierzu muss zunächst bemerkt werden, dass, wie bereits ausgeführt, diese Leitlinien und Mitteilungen - von Ausnahmen abgesehen - keinen Rechtsnormcharakter haben. Sie beschreiben lediglich die Vorgehensweise der Kommission bei der Prüfung der Zuwiderhandlung und die Kriterien, zu deren Berücksichtigung sie sich verpflichtet

17 Vgl. z.B. Jürgen Schwarze: Europäische Kartellbußgelder im Lichte übergeordneter Vertrags- und Verfassungsgrundsätze. In: Europarecht, 2009, S.171 ff.; Wolfgang Weiß:Das Leitlinien(un)wesen der Kommission verletzt den Vertrag von Lissabon. In: Europäisches Wirtschafts- und Steuerrecht, 2010, Heft 7, S.257 ff.

18 Vgl. Ralph Alexander Lorz: Das Problem des demokratischen Defizits. In: Die Unionsordnung. Handbuch zur Europäischen Verfassung. Hrsg. v. Dimitris Th. Tsatsos. Berlin 2010, S.331 ff.

19 Vgl. Christoph Vedder in Vedder, Heintschel von Heinegg (Hrsg.): Europäischer Verfassungsvertrag. Baden-Baden 2007, Art.I-36, Rdnr.2. 
hat. ${ }^{20}$ Selbstverpflichtungen einer Behörde sind insoweit interne Maßnahmen, die, auch wenn sie keine rechtliche Regelung enthalten, auch rechtliche Außenwirkungen erzeugen können, in dem sie Vertrauen der Allgemeinheit auf ein bestimmtes Verhalten der Kommission erzeugen. Sie können deshalb als „Handlungen allgemeinen Charakters“ Gegenstand einer Klage vor den Gerichten der Union sein. ${ }^{21}$

Doch wegen des fehlenden Rechtsnormcharakters bedeutet die gerichtliche Überprüfung der Leitlinien und Mitteilungen rechtsdogmatisch keine Überprüfung einer Rechtsnorm wie z.B. einer Verordnung oder Direktive. Genau betrachtet, prüfen die Gerichte, ob das in den Leitlinien und Mitteilungen beschriebene Verhalten der Kommission den rechtsstaatlichen Anforderungen entspricht, und oder ob das tatsächliche Verhalten der Kommission dem dort beschriebenen (also „versprochenen“) Verhalten entspricht, d.h., ob die Kommission im Falle der Abweichung das Vertrauenschutzprinzip verletzt hat.

Das gemäß den Leitlinien und Mitteilungen ,vorausbestimmte“ Verhalten der Kommission muss aber nach den rechtlichen Bestimmungen beurteilt werden, die zum Zeitpunkt des entsprechenden Verhaltens gültig sind, denn es kommt auf das Verhalten und nicht auf die entsprechende interne Maßnahme - Leitlinie oder Mitteilung - an. Daraus folgt, dass das in den Leitlinien und Mitteilungen beschriebene Verhalten der Kommission nach dem Inkrafttreten der Lissaboner Vertrages gemäß den neuen vertraglichen Bestimmungen, also auch nach Art. 290 Abs. 1, zu beurteilen ist.

\section{Erfüllung der Voraussetzungen des Art. 290 Abs. 1?}

Würde man der hier vertretenden Auffassung folgen, dass die Bußgeldverhängungspraxis der Kommission auf Sachverhalte nach dem Inkrafttreten des Vertrags von Lissabon den Anforderungen des Art. 290 Abs. 1 AEUV entsprechen muss, fragt es sich, ob die oben aufgezeigte „Gesamtkonstruktion“ der Rechtsgrundlage für die Festsetzung der Bußgelder, die aus der Regelung der Art. 2 und 3 der Verordnung Nr.1/2003 und den diese klarstellenden Leitlinien und Mitteilungen bestehen, den im Vergleich zur früheren Rechtslage deutlicher herausgestellten Anforderungen an das Demokratieprinzip im Art. 290 Abs. 1 AEUV entspricht.

Danach müsste gemäß Art. 290 Abs. 1 UAbs. 2 S.2 AEUV das „Wesentliche“ des Bußgeldfestetzungsverfahrens" in der Rechtsverordnung Nr.1/2003 selbst geregelt worden sein. Dazu kann man nur die in Art. 23 Abs. 2 und 3 der Verordnung Nr.1/2003 tatsächlich enthaltenen Regelungen zählen - nämlich die Regelung, dass die Höhe des Bußgeldes ist auf $1 \%$ des Gesamtumsatzes im vorausgegangenen Geschäftsjahr für leichte Verstöße und auf $10 \%$ für andere Verstöße beschränkt bleibt und dass bei der Bemessung der Geldbuße die Dauer und die Schwere des Verstoßes zu berücksichtigen ist.

Die Leitlinien und Mitteilungen - in ihrer Bedeutung für die konkrete Ausgestaltung des Verfahrens - können hier als „ergänzende Regelungen“ aber nicht berücksichtigt werden - nicht nur, weil sie mangels Rechtsnormcharakters kein „Rechtsakt ohne Gesetzescharakter mit allgemeiner Geltung“ gem. Art. 290 Abs. 1 UAbs. 1 AEUV sind,

20 Das Urteil der EuGH in der Rechtssache C-386/10 P v. 8.12.2011, Chalkor, §60.

21 Vgl. das Urteil des EuGH in der Rechtssache C-171/00 P v. 15. 1. 2002, Libéros, §35. 
sondern weil es bereits an einer Vorschrift zur Befugnisübertragung an die Kommission zu ihrem Erlass gem. Art. 290 Abs. 1 UAbs. 1 und UAbs. 2 S.1 AEUV fehlt.

Die Leitlinien könnten aber bei der Regelung des Art. 23 Abs. 2 und 3 der Verordnung Nr.1/2003 zwar nicht als ,ergänzende Regelungen“, aber als das, was sie sind - nämlich Selbstverpflichtungen zur Konkretisierung des der Kommission eingeräumten weiten Ermessens - „mitgelesen werden“. Denn aus Art. 290 Abs. 1 AEUV ergibt sich nicht, dass nach dem Inkrafttreten des Vertrags von Lissabon eine Einschränkung des durch Gesetzgebungsakte der Kommission eingeräumten Ermessens im Interesse der Rechtssicherheit durch Leitlinien und Mitteilungen ohne Rechtsnormcharakter nicht mehr möglich wäre. Eine solche Möglichkeit auszuschließen war weder die Absicht bei der Einführung des Art. 290 AEUV, noch wäre ein solcher Ausschluss sinnvoll.

Voraussetzung ist nur, dass Leitlinien und Mitteilungen im Rahmen des der Kommission eingeräumten Ermessen bleiben. Überschreiten solche Selbstverpflichtungen aber die Grenzen des durch Ermächtigungsgrundlage eingeräumten Ermessens, so sind sie nicht mehr nur interne Maßnahmen, sondern stellen bereits eine rechtliche „Regelung“" dar, die mangels Ermächtigung nicht in der Kompetenz der Kommission liegt und somit nichtig wäre. ${ }^{22}$

Hier stellt sich die Frage, ob der Unionsgesetzgeber in Art. 23 Abs. 2 und 3, aber auch in Abs. 4 der Verordnung Nr.1/2003 selbst alles „Wesentliche“ zum Verfahren der Bußgeldfestsetzung geregelt hat. Dabei dürfen der Kommission durchaus Ermessenspielräume bleiben, allerdings nur bei der Anwendung der vom Unionsgesetzgeber getroffenen „,wesentlichen“ Regelungen - nicht aber bei der Regelung von ,unwesentlichen" Fragen, denn dafür braucht die Kommission eine ausdrückliche Ermächtigung des Unionsgesetzgebers gem. Art. 290 Abs. 1 AEUV, welche hier nicht vorliegt.

Die Frage, die hier zu beantworten ist, lautet somit: Hat die Kommission mit der Verpflichtung in den Leitlinien und Mitteilungen, sich auf eine bestimmte Weise zu verhalten, sich nur im Rahmen des ihr im Art. 23 der Verordnung Nr.1/2003 eingeräumten Ermessens die dort getroffenen ,wesentlichen“ Regelungen nur - zulässigerweise - angewendet, oder bereits - unzulässigerweise - zusätzliche Regelungen getroffen?

Bei einer „natürlichen“ Sichtweise liegt es auf der Hand, dass die Leitlinien und Mitteilungen neue, zusätzliche Regelungen zu der inhaltlich dürftigen Regelung des Art. 23 - ob ,wesentlich“ oder nicht - geschaffen haben. Damit hätte sich die Kommission unzulässigerweise zum „Ersatzgesetzgeber“ aufgeschwungen. ${ }^{23}$

Man könnte aber die Frage stellen, ob dann, wenn die Kommission keine entsprechenden Leitlinien und Mitteilungen verfasst, aber bei der Festsetzung der Bußgelder ständig so verfahren wäre, wie dort beschrieben, insbesondere die Bußgelder in der in den Leitlinien wiedergegebenen Weise berechnet und die „Kronzeugen“ privilegiert hätte, ein solches Verfahren noch im Rahmen des ihr vom Art. 23 der Verordnung Nr. 1/2003 eingeräumten Anwendungsermessens gelegen hätte (bei Außerachtlassung des Problems der Rechtssicherheit und Transparenz).

22 Vgl. Jürgen Bast: Handlungsformen. In: Armin von Bogdandy (Hrsg.): Europäisches Verfassungsrecht. Berlin 2003, S.518.

23 So z.B. Wolfgang Weiß: Lissabon und der Vollzug des EU-Wettbewerbsrechts. In: Europäisches Wirtschafts- und Steuerrecht. 2011, Heft 1-2. 
Der EuGH hat bisher die Frage der Gültigkeit der Rechtsgrundlage des Art. 23 der Verordnung Nr.1/2003 für das Bußgeldverfahren positiv beantwortet und die Leitlinien und Mitteilungen nur als Selbstverpflichtungen der Kommission zur Ermessensausübung im Dienst der Rechtssicherheit, also noch als innerhalb des Anwendungsermessens liegend, qualifiziert. ${ }^{24}$ Obwohl das Wesentlichkeitsprinzip auch vor dem Vertrag von Lissabon im Unionsrecht galt, ${ }^{25}$ hat der EuGH das in Art. 23 Abs. 2 und 3 der Kommission eingeräumte weite Ermessen bei der Anwendung dieser Vorschrift nicht als ein Problem gesehen. ${ }^{26}$

Freilich haben die Unionsgerichte noch nicht die Gelegenheit gehabt, sich mit der Rechtslage nach dem Inkrafttreten des Vertrages von Lissabon und damit diese Frage unter dem Gesichtspunkt des neuen Art. 290 Abs. 1 AEUV zu prüfen. Wie bereits ausgeführt, kodifiziert diese Vorschrift den Gesetzesvorbehalt und das Wesentlichkeitsprinzip und verleiht diesen deutlichere Konturen als zuvor.

Kann aus dem neuen Art. 290 Abs. 1 AEUV der Schluss gezogen werden, dass der Wille des Vertrages auf die Verschärfung des Prüfungsmaßstabs des Demokratieprinzips gerichtet ist? Und wird der eventuell verschärfte Prüfungsmaßstab zu einem anderen Ergebnis bei der Beurteilung der Frage führen, ob das durch Art. 23 Abs. 2 und 3 der Verordnung Nr.1/2003 der Kommission eingeräumte weite Anwendungsermessen noch die Gegenstände umfasst, die als Selbstverpflichtungen der Kommission erst in den Leitlinien und Mitteilungen zum Vorschein kommen, oder sind diese so ,wesentlich“, dass sie bereits in der Verordnung selbst (also vom Unionsgesetzgeber) geregelt werden sollten? Bei der Suche nach der Antwort kann auf zwei Aspekte hingewiesen werden.

Erstens, bei der Frage, „was ist wesentlich“, handelt es sich um eine Wertungsfrage, bei der nicht nur das Prinzip der Autonomie des Unionsrechts zu berücksichtigen ist, sondern auch die nationalen Traditionen der Mitgliedstaaten eine Rolle spielen. Hinsichtlich der Bedeutung und der Anforderungen des Gesetzesvorbehalts und des Wesentlichkeitsprinzips sind die nationalen Traditionen der Mitgliedstaaten aber verschieden. Dabei gehören Deutschland und neuerdings auch die neuen Mitgliedstaaten zu derjenigen Gruppe, die ein hohes Maß an Verrechtlichung des Verwaltungsrechts (Verwaltungsverfahrensgesetze) aufweisen und die dem Gesetzesvorbehalt und dem Wesentlichkeitsprinzip eine höhere Bedeutung beimessen. Auf der anderen Seite stehen z.B. Frankreich und die von ihm beeinflussten Systeme, die (noch) kein weitgehend gesetzlich kodifiziertes Verwaltungsrecht haben, und wo viele verwaltungsrechtliche Figuren sich nicht im Gesetz, sondern in der Rechtsprechung und in von der Verwaltung herausgegeben Vorschriften finden lassen und die traditionell der Verwaltung größere Gestaltungsspielräume zugestehen, ohne darin sogleich eine Verletzung des Demokratieprinzips zu sehen.

24 Deutlich im Urteil der EuGH in der Rechtssache C-266/06 v. 28.5.2008, Evonik Degussa, $\S \S 38$ ff.; 57 ff.; vgl. dazu auch Peter Whelan: The Degussa case. The Court of First instance and the European Court of Justice have taken clear line on legal certainty and antitrust fines. In: Competition Law Insight, 29 July 2008, p.13 ff.

25 Ines Härtel: Handbuch Europäische Rechtsetzung. Berlin 2006, S.236 ff.

26 Peter Whelan: The Degussa case. The Court of First instance and the European Court of Justice have taken clear line on legal certainty and antitrust fines. In: Competition Law Insight, 29 July 2008, p.13 ff., p.15. 
Zweitens, es kann trotz dieser Divergenz eine allgemeine Tendenz bzw. Forderung kaum übersehen werden, die auf eine stärkere rechtliche Eingrenzung des Ermessens der Verwaltung und auf die Höherstufung der Regelungen von internen Verwaltungsmaßnahmen zu gesetzlichen Regelungen gerichtet ist. Die in den letzten zwei Dekaden in vielen Mitgliedstaaten erstmals verabschiedeten Gesetze zur Regelung verschiedener Aspekte des Verwaltungsverfahrens könnten als Beleg dafür angeführt werden. Man könnte verleitet sein, die Auffassung zu vertreten, auch die Einfügung des neuen Art. 290 Abs. 1 im Vertrag, der dem Gesetzesvorbehalt und dem Wesentlichkeitsprinzip deutlichere Konturen gibt, ist ein Ausdruck dieses Denkens, und bedeutet nicht nur eine reine Kodifizierung der bisherigen Rechtslage, sondern stellt eine gewisse qualitative „Höherstufung“ des Demokratieprinzips, also eine Stärkung der demokratischen Legitimation der Union dar.

Ob das der Fall ist, muss noch geklärt werden. Auf jeden Fall werden die Unionsgerichte wohl bald damit befasst sein. ${ }^{27}$

27 Klage v. 2.10.2010 in der Rechtssache T-364/10, Duravit, Abl. C, 288, 23.10.2010, S.59. 